\title{
Prácticas evaluativas de personal docente de educación especial para estudiantado con discapacidad intelectual, integrado en escuelas regulares
}

\author{
Evaluative Practices of Teachers of Special Education for Students with Intellectual \\ Disabilities integrated into Regular Schools
}

\section{Práticas de avaliação do corpo docente de educação especial para crianças em idade escolar com deficiência intelectual, integradas em escolas regulares}

\author{
Marcela Bizama \\ Universidad Católica de la Santísima Concepción \\ Concepción, Chile \\ mbizama@ucsc.cl \\ https://orcid.org/0000-0003-3515-4504 \\ Joanna Martínez \\ Universidad Católica de la Santísima Concepción \\ Concepción, Chile \\ joannacarol@gmail.com \\ https://orcid.org/0000-0001-7457-2077
}

Recibido • Received • Recebido: 26 / 10 / 2019

Corregido • Revised • Revisado: 30 / 06 / 2021

Aceptado • Accepted • Aprovado: 02 / 08 / 2021

\section{Resumen}

Objetivo. El propósito de esta investigación fue conocer las prácticas evaluativas diferenciadas que realiza el profesorado chileno de educación especial para estudiantes que presentan necesidades educativas especiales derivadas de discapacidad intelectual, quienes se integran en escuelas de educación general básica a través de los Proyectos de Integración Escolar (PIE). El estudio pretendió, además, analizar los significados que los equipos docentes de educación especial atribuyen a sus propias prácticas de evaluación. Metodología. La investigación se realizó desde un enfoque metodológico cualitativo con un diseño de estudio de caso múltiple. Las personas participantes fueron 10 docentes de educación especial en servicio, de 9 establecimientos educacionales diferentes de la región del Biobío, Chile. El instrumento utilizado para la recopilación de información fue la entrevista semiestructurada. La información recogida fue analizada a través de la técnica de análisis de contenido temático. Resultados. Los hallazgos muestran que los educadores y las educadoras especiales que se entrevistaron utilizan procedimientos de evaluación diferenciada alineados con los principios de la evaluación general, pero con una participación incipiente de elementos propios de la especialidad, para dar respuesta a las necesidades educativas especiales del estudiantado que presenta discapacidad intelectual. Conclusiones. Se concluye que es necesario que los centros reflexionen sobre los procesos de enseñanza y prácticas de evaluación que llevan a cabo, con el fin de establecer mejoras en sus dinámicas y procesos de aprendizaje del estudiantado con discapacidad intelectual que asiste a centros regulares de enseñanza.

Palabras claves: Educación especial; evaluación diferenciada; necesidades educativas especiales; discapacidad intelectual; prácticas evaluativas; adecuaciones curriculares. 
http://doi.org/10.15359/ree.25-3.22

http://www.una.ac.cr/educare

educare@una.ac.cr

\begin{abstract}
Objective. The purpose of this research was to know the differentiated evaluation practices implemented by the special education teachers for students with special educational needs deriving from intellectual disability and are integrated into basic general education schools through the School Integration Projects (PIE). The research also analyzed the meanings that the special education teachers attribute to their own practices. Method. The research was conducted under a qualitative approach with a multiple case study design. The participants were 10 Special Education teachers in service from nine different educational establishments. The instrument used to gather information was a semistructured interview. The information was analyzed through the thematic content analysis technique. Results. The findings show that most of the differential educators interviewed perform differentiated evaluation procedures for students with intellectual disability; these procedures are aligned with the principles of the general evaluation in order to respond to the special educational needs of the students. Conclusions. It is concluded that the centers need to conduct a reflexive analysis of teaching processes and evaluation practices that they implement in order to establish improvements in their dynamics in favor of students with intellectual disabilities who attend regular teaching centers.
\end{abstract}

Keywords: Special educational needs; intellectual disability; special evaluation; evaluative practices; curricular adaptations.

\begin{abstract}
Resumo
Objetivo. O objetivo desta pesquisa foi conhecer as práticas diferenciadas de avaliação realizadas pelo corpo docente de Educação Especial para estudantes integrados nas escolas de educação básica, que apresentam necessidades educacionais especiais derivadas de deficiência intelectual, por meio dos Projetos de Integração Escolar (PIE). Outro propósito dessa pesquisa também foi analisar os significados que o corpo docente de Educação Especial atribue às suas próprias práticas de avaliação. Metodología. A pesquisa foi conduzida sob uma abordagem metodológica qualitativa, com um desenho de estudo de caso múltiplo. Participaram 10 professores de Educação Especial de nove diferentes estabelecimentos de ensino. $O$ instrumento utilizado para coletar informações foi a entrevista semiestruturada. As informações foram analisadas pela técnica de análise de conteúdo temático. Resultados. Os resultados mostram que a maioria dos educadores especiais entrevistados realiza procedimentos de avaliação diferenciados para os estudantes que apresentam deficiência intelectual, alinhados aos princípios da avaliação geral, com o objetivo de responder suas necessidades educacionais especiais. Conclusões. Conclui-se que é necessário que os centros realizem uma análise reflexiva dos processos de ensino e práticas de avaliação que realizam, a fim de estabelecer melhorias em sua dinâmica em favor dos estudantes com deficiência intelectual que frequentam os centros regulares de ensino.
\end{abstract}

Palavras-chave: Avaliação; avaliação diferencial; necessidades educativas especiais; deficiência intelectual; práticas avaliativas; ajustes curriculares.

\title{
Introducción
}

Según de Vries (2015), la evaluación en la educación se introdujo hace varias décadas como un eje importante de las reformas educativas y ha sido impulsada por la mayoría de los gobiernos del mundo. En ese sentido, como indica también Rueda Beltrán (2010), la evaluación educativa es una actividad pedagógica que se ha tornado una tarea altamente compleja y que 
http://doi.org/10.15359/ree.25-3.22

ha requerido cada vez más de docentes con formación especializada y conocimiento acumulado consistente. En efecto, tal como señalan Barría Rojas y Jurado de los Santos (2016), las prácticas educativas se basan en conocimientos técnicos. Estos saberes pedagógicos deberían orientar, entonces, la actuación del profesorado en el ámbito de la Educación para Todos, cuyo texto fue aprobado en el Foro Mundial sobre la Educación realizado en Dakar en el año 2000.

En relación con lo anterior, uno de los mayores desafíos a nivel educacional internacional actual, según Arnaiz Sánchez et al. (2015), es la inclusión educativa de necesidades educativas especiales (NEE) en las escuelas regulares de enseñanza, puesto que, en el escenario educativo formal, uno de los puntos de inflexión más importante es la evaluación de los aprendizajes de todo el estudiantado (Román y Murillo, 2013).

Respecto de la evaluación de aprendizajes, una de las especialidades de la evaluación educativa, se trata de un constructo polisémico, complejo y multimodal que puede tener diferentes significados, ser usado en distintos sentidos y, por ende, puede generar controversia, tanto en su conceptualización como en su utilización.

¿Cómo se evalúa actualmente en las escuelas regulares a las escolares y los escolares integrados que presentan necesidades educativas especiales (NEE) derivadas de discapacidad intelectual (DI), en el marco de las nuevas orientaciones internacionales de integración/inclusión escolar? En la bibliografía especializada de los últimos años, se han reportado algunos estudios internacionales y nacionales al respecto.

A nivel internacional, el estudio de Spooner et al. (2017) señala que, en USA, luego de la Ley No Child Left Behind de 2001, que continuó con la Ley de Éxito de Todos los Estudiantes (ESSA), toda la niñez, incluida aquella con discapacidades severas, debe aprender habilidades de vida diaria y académicas utilizando prácticas basadas en evidencia.

En América Latina, los procesos evaluativos destinados a escolares con NEE han sido objeto de estudio en diversos países. Agostini Martins et al. (2018) realizan un análisis de los procesos evaluativos y de las modalidades de diagnóstico en Argentina y Brasil. En ese trabajo, se reportan las prácticas evaluativas utilizadas para la identificación y la derivación de niñez a los establecimientos educacionales de apoyo escolar y a los servicios asistenciales existentes en ambos países.

En Chile, el trabajo de Calderón Bravo (2011) analizó los discursos y prácticas de evaluación diferenciada de docentes de enseñanza básica de tres establecimientos educacionales diferentes de Santiago de Chile, región Metropolitana, que atienden a escolares con NEE en las aulas regulares. El estudio muestra que el personal docente de educación general básica no tienen conocimientos técnicos ni políticos acerca de la evaluación diferenciada que deberían aplicar al estudiantado con NEE y que no se rigen por modelos teóricos de evaluación especial, sino solo por su práctica y experiencia profesional personal. 
http://doi.org/10.15359/ree.25-3.22

http://www.una.ac.cr/educare

educare@una.ac.cr

Otro estudio nacional, llevado a cabo por Jara Henríquez y Jara Coatt (2018) en un mismo establecimiento educacional de la ciudad deTalcahuano, región del Biobío, Chile, concluye que el profesorado participante de la investigación realiza adecuaciones curriculares o modificaciones a ciertos elementos del currículo para que el estudiantado con NEE derivadas de discapacidad puedan avanzar en su aprendizaje, sin embargo, tanto sus concepciones sobre evaluación como sus prácticas evaluativas se soportan en el enfoque tradicional de la evaluación.

\section{Evaluación de aprendizajes: Tipos, procedimientos e instrumentos}

Desde el punto de vista de los momentos de aplicación de la evaluación, así como de su intencionalidad, se distinguen 3 tipos de evaluación de aprendizajes: inicial, procesual y final.

La evaluación inicial, entendida también como evaluación diagnóstica, se realiza al comienzo de algún ciclo académico, de un proceso o de una implementación de un programa educativo y permite la recogida de datos en el momento de partida, de modo que el profesorado o personal docente inicie el proceso conociendo a cabalidad la situación inicial de aprendizaje del estudiantado.

La evaluación procesual, por su parte, tiene como función ser formativa y brinda información continua y sistemática del proceso educativo a lo largo de un periodo determinado, con el fin producir mejoras en el proceso que lleva a cabo durante el aprendizaje. Este tipo de evaluación permite tomar decisiones durante el proceso de enseñanza-aprendizaje, porque se va obteniendo constantemente información del desarrollo del aprendizaje del estudiantado, lo que permite regular, orientar, modificar, retomar o modificar el proceso educativo que se está realizando (Muñoz Vega, 2006).

La evaluación final, permite acreditar los aprendizajes logrados a través de la recogida de datos o resultados al final de un periodo de tiempo determinado para la realización de un programa o el logro de un aprendizaje (Cajigas y García, 2014; Muñoz Vega, 2006).

Por otra parte, los procedimientos evaluativos o prácticas evaluativas son todas aquellas acciones referidas a la evaluación de los aprendizajes del estudiantado. Santibáñez Riquelme (2001) las define como "todo aquel medio y recurso -estructurado con rigor científico- que permite al docente recabar informaciones directas o indirectas, pero sistemáticas, sobre los comportamientos estudiantiles, y están destinadas a favorecer una oportuna y adecuada toma de decisiones relativas a un hecho educativo" (p. 53). Estos pueden ser de tipo formal, semiformal o informal. Las técnicas o procedimientos formales se refieren a aquellos en los cuales existe una planeación que tiene reglas o normas; generalmente se asocian a una evaluación de tipo cuantitativa o cualitativa y se consideran para esta instancia instrumentos tales como: pruebas escritas, orales o de ejecución, test, listas de cotejos y escalas de diversos tipos. Los procedimientos semiformales, en cambio, son aquellos referidos a ejercicios realizados en clases, fuera de clases, así como desempeños o trabajos grupales. Este tipo de evaluación requiere mayor tiempo de preparación y mayor tiempo para la valoración. Se puede o no 
http://doi.org/10.15359/ree.25-3.22

generar calificación a partir de ellos. Finalmente, las técnicas o procedimientos informales son breves y se realizan a través de actos pedagógicos tales como la observación y la exploración a través de preguntas. Generalmente no conducen a calificación.

Los tipos de instrumentos de evaluación, por su parte, se refieren a los medios con los cuales se obtendrá la información respecto del aprendizaje del estudiantado. Desde esta perspectiva, son registros con características propias, que sirven para recoger la información requerida en función de las características del aprendizaje que se pretende evaluar. Asimismo, todo instrumento de evaluación debería especificar las condiciones en las que se debe aplicar. Para Castillo Arredondo y Cabrerizo Diago (2003), el instrumento evaluativo es el recurso necesario que se utiliza con una técnica concreta. Los instrumentos de evaluación se pueden clasificar o diseñar también, de acuerdo con el momento de aplicación o según su finalidad, distinguiéndose: instrumentos para la evaluación inicial o diagnóstica, instrumentos para la evaluación procesual o formativa e instrumentos para la evaluación final o sumativa.

\section{Necesidades educativas especiales y evaluación diferenciada}

La educación especial es definida en Chile como aquella modalidad del sistema escolar que provee servicios y recursos especializados, tanto a los establecimientos de enseñanza regular como a las escuelas especiales, "con el propósito de asegurar, de acuerdo a la normativa vigente, aprendizajes de calidad a niños, niñas, jóvenes ... con necesidades educativas especiales (NEE), de manera que accedan, participen y progresen en el currículum nacional en igualdad de condiciones y oportunidades [Ley General de Educación y Ley 20.422]" (Ministerio de Educación de Chile [MINEDUC], párr. 1, 2016).

Por otra parte, las NEE, son todas aquellas necesidades educativas que no pueden ser resueltas a través de los medios y recursos metodológicos tradicionales que se utilizan habitualmente en las aulas para todo el alumnado (MINEDUC, 2005). De este modo, dichas necesidades educativas deben ser atendidas por personal educador diferencial o especial, para responder a las diferencias individuales de la diversidad del estudiantado presente en las aulas. Para ello, resulta necesario incorporar ajustes, recursos y medidas pedagógicas especiales o de carácter extraordinario, generalmente distintas a las utilizadas para el común de sus pares del mismo curso que no presentan NEE.

Las NEE se clasifican en NEE permanentes y en NEE transitorias (MINEDUC, 2009). Son necesidades educativas permanentes, aquellas derivadas de discapacidad intelectual, trastorno motor, espectro autista, disfasia, discapacidad visual o discapacidad auditiva. Las NEE permanentes perduran a lo largo del ciclo vital del estudiante. Por otro lado, se clasifican como necesidades educativas especiales transitorias, aquellas que se presentan en un momento o etapa del proceso educativo del estudiante y son derivadas de dificultades específicas del lenguaje o dificultades específicas del aprendizaje. Desde esa perspectiva, la DI constituye, entonces, una NEE permanente. 
http://doi.org/10.15359/ree.25-3.22

http://www.una.ac.cr/educare

educare@una.ac.cr

En Chile, se entiende por evaluación diferenciada a un procedimiento pedagógico que permite al personal docente identificar los niveles de logro de los aprendizajes que alcanzan a nivel curricular aquel estudiantado que, por diferentes NEE, está en una situación distinta a la mayoría de sus pares, de manera transitoria o permanente (MINEDUC, 1997).

Es así como, el Decreto de Evaluación N.5 511 del Ministerio de Educación de Chile que aprueba el Reglamento de Evaluación y Promoción Escolar de niños y niñas de Enseñanza Básica, en su artículo N. 5 establece: "A los alumnos que tengan impedimentos para cursar en forma regular un subsector, asignatura o actividad de aprendizaje, deberá aplicárseles procedimientos de evaluación diferenciada" (MINEDUC, 1997, De la Evaluación, párr. 4).

Desde esa perspectiva, entonces, cabe preguntarse: ¿Cuáles son las prácticas evaluativas diferenciadas del profesorado de educación especial que trabajan en establecimientos comunes de enseñanza para escolares con NEE derivadas de DI?

En relación con lo anteriormente expuesto, esta investigación se planteó como principal propósito conocer las prácticas evaluativas diferenciadas o adaptadas más comunes que utiliza el profesorado de educación especial para escolares que presentan DI que asisten a Proyectos de Integración Educativa (PIE) en establecimientos de Educación General Básica, así como develar los significados que el personal docente le otorga a sus propias prácticas.

\section{Método}

\section{Diseño}

Se utilizó un enfoque cualitativo basado en el paradigma comprensivo-interpretativo y un diseño de estudio de caso múltiple (Stake, 2010), puesto que lo que interesaba era conocer las prácticas evaluativas del profesorado de educación especial en tanto que actores de la educación general y especial desde su propia perspectiva, considerando que el contexto escolar es un factor constituido por los significados que la comunidad le atribuye (Sandín Esteban, 2003; Tójar Hurtado, 2006).

\section{Participantes}

Las personas participantes fueron 10 docentes de educación especial en servicio, que se desempeñan en los Programas de Integración Escolar (PIE) de 9 establecimientos diferentes de la Comuna de Coronel, región del Biobío, Chile, que trabajan con estudiantado que presenta NEE derivadas de $\mathrm{DI}$ en grado leve o moderado. Todas estas personas cuentan con título profesional de educador diferencial otorgado por una universidad nacional y tenían entre 3 y 25 años de servicio profesional en el momento de la investigación. El tamaño de la muestra definitiva dependió de la saturación horizontal de la información obtenida (Hammersley y Atkinson, 1994). Las características del equipo de docentes participantes del estudio se visualizan en la Tabla 1.

6 
Tabla 1: Características de las personas participantes del estudio

\begin{tabular}{lcllc}
\hline Caso & $\begin{array}{c}\text { Establecimiento } \\
\text { educacional }\end{array}$ & Sexo & Título profesional & $\begin{array}{c}\text { Años de servicio } \\
\text { profesional }\end{array}$ \\
\hline Entrevistada 1 & 1 & Mujer & Educadora diferencial & 4 años \\
Entrevistado 2 & 2 & Hombre & Educador diferencial & 7 años \\
Entrevistada 3 & 3 & Mujer & Educadora diferencial & 6 años \\
Entrevistada 4 & 4 & Mujer & Educadora diferencial & 25 años \\
Entrevistada 5 & 5 & Mujer & Educadora diferencial & 8 años \\
Entrevistada 6 & 6 & Mujer & Educadora diferencial & 12 años \\
Entrevistad 7 & 7 & Mujer & Educadora diferencial & 14 años \\
Entrevistada 8 & 1 & Mujer & Educadora diferencial & 18 años \\
Entrevistada 9 & 8 & Mujer & Educadora diferencial & 8 años \\
Entrevistada 10 & 9 & Mujer & Educadora diferencial & 3 años \\
\hline
\end{tabular}

Nota: Elaboración propia.

\section{Instrumento}

Para la recolección de datos se utilizó una entrevista semiestructurada, validada a través del procedimiento de criterio experto y cuyo guion consideró las categorías consignadas en la Tabla 2.

Tabla 2: Categorías deductivas para el análisis del corpus discursivo

\begin{tabular}{ll}
\hline \multicolumn{1}{c}{ Categoría } & \multicolumn{1}{c}{ Definición } \\
\hline Tipos de evaluación & $\begin{array}{l}\text { Tipos de evaluación aplicados para el estudiantado que presenta discapacidad } \\
\text { intelectual. }\end{array}$ \\
Procedimientos evaluativos & $\begin{array}{l}\text { Procedimientos evaluativos más comunes utilizados en las prácticas evaluativas } \\
\text { para el estudiantado con discapacidad intelectual integrados en PIE en los } \\
\text { establecimientos participantes. }\end{array}$ \\
Instrumentos de evaluación & $\begin{array}{l}\text { Instrumentos utilizados para evaluar los aprendizajes del estudiantado con } \\
\text { discapacidad intelectual. }\end{array}$ \\
\hline
\end{tabular}

Nota: Elaboración propia. 
http://doi.org/10.15359/ree.25-3.22

http://www.una.ac.cr/educare

educare@una.ac.cr

\section{Procedimiento y aspectos éticos}

Se firmaron consentimientos informados con cada una de las personas participantes del estudio. Las entrevistas cara a cara fueron realizadas en forma individual, en dependencias escolares facilitadas por los establecimientos educacionales respectivos y tuvieron una duración aproximada de 45 minutos. Se realizó una grabación de audio para cada una de ellas, lo que permitió su posterior transcripción y análisis.

\section{Técnicas de análisis de la información}

Una vez recopilada la información se la sintetizó y organizó en unidades manejables, buscando regularidades o modelos y constatando aquello importante que diera respuesta a las interrogantes del estudio. Para el análisis de la información recopilada, se aplicóla técnica de análisis de contenido temático, que implica captar el contenido latente de la comunicación y, por tanto, busca una interpretación del significado latente del discurso (Krippendorff, 1990). Con respecto al proceso de codificación de la información, este se llevó a cabo agrupando la información obtenida en categorías temáticas (Fernández Núñez, 2006), las que se organizaron en 3 dimensiones: tipos de evaluación, procedimientos e instrumentos. Coherentemente con ello, se utilizaron matrices de análisis según las categorías previamente definidas en el guion de entrevista.

\section{Resultados}

Los resultados se presentan a continuación, organizados de acuerdo con las categorías de análisis utilizadas en el procesamiento de la información recopilada a través de las entrevistas individuales y de los objetivos del estudio.

Tipos de evaluación que realizan los educadores y las educadores diferenciales, para el estudiantado que presenta discapacidad intelectual que asisten a PIE en escuelas regulares

Como ya señalamos anteriormente, los tipos de evaluación corresponden a la clasificación de las evaluaciones utilizadas por el personal docente en su quehacer evaluativo. Desde el punto de vista de los momentos de aplicación de la evaluación, así como de su intencionalidad, se distinguen 3 tipos: evaluación inicial o diagnóstica, evaluación procesual y evaluación final.

Al respecto, los grupos profesionales de educación especial entrevistados dicen realizar evaluación diagnóstica a inicios del año escolar. Por otra parte, realizan evaluación procesual, durante el proceso de enseñanza aprendizaje y evaluación final, antes del término del año lectivo, alineándose, de esta manera, con los principios de la evaluación general de la educación regular. 
http://doi.org/10.15359/ree.25-3.22

Respecto de la evaluación inicial o diagnóstica, los significados construidos en el discurso del personal docente entrevistado están referidos a que este tipo de evaluación es de mucha importancia, puesto que permite al personal docente especialista comenzar el año escolar conociendo el nivel de desarrollo y el nivel de aprendizaje de sus estudiantes con DI. En relación con la evaluación procesual, tal como señalan en sus discursos, esta favorece la toma de decisiones durante el proceso de enseñanza-aprendizaje y la posibilidad de replantear las prácticas. Por otra parte, la evaluación final les permite, a fines del año escolar, evaluar los avances del estudiantado. Algunas de las respuestas que ilustran esta dimensión, pueden visualizarse en la Tabla 3.

Tabla 3: Tipos de evaluación realizada por educadores diferenciales para estudiantes que presentan discapacidad intelectual que asisten a PIE en escuelas regulares

\begin{tabular}{cl}
\hline Participante & \multicolumn{1}{c}{ Contenido declarado } \\
\hline Participante 4 & $\begin{array}{l}\text { Los tipos de evaluación que se realizan para evaluar ... a estudiantes con discapacidad intelectual } \\
\text { son los siguientes: se inicia con evaluación diagnóstica siendo esta la principal, se continúa con las } \\
\text { evaluaciones de proceso que es la evaluación que me permite el monitoreo de sus aprendizajes y la } \\
\text { toma de decisiones para replantear mis prácticas, con el fin de ayudar al estudiantado a conseguir } \\
\text { los objetivos propuestos en su adecuación curricular. }\end{array}$ \\
Participante 8 & Los tipos de evaluación como la inicial, evaluaciones de proceso, evaluación final. Esas. \\
Participante 9 & $\begin{array}{l}\text { Utilizo los tipos de evaluación: inicial, el diagnóstico ¿cierto?, las evaluaciones de proceso, para verificar } \\
\text { los aprendizajes durante el año escolary la evaluación final, para ver si el alumnado avanzó o no. }\end{array}$ \\
\hline
\end{tabular}

Nota: Elaboración propia.

\section{Procedimientos más comunes de evaluación diferenciada utilizados por los educadores y las educadoras diferenciales, para el estudiantado con discapacidad intelectual que asiste a PIE en escuelas regulares de enseñanza}

Como se señaló anteriormente, los procedimientos de evaluación diferenciada corresponden a las acciones que realizan los educadores y las educadoras de educación especial o diferencial para dar respuesta al proceso evaluativo del estudiantado que presenta DI y que se encuentran integrados en los proyectos PIE en establecimientos regulares de enseñanza en Chile, conforme a los lineamientos actuales de la política nacional.

La mayoría de quienes participaron del estudio afirma utilizar procedimientos de evaluación diferenciada a través de adecuaciones a los instrumentos comunes de evaluación utilizados por el profesorado de educación general básica, adaptándolos y utilizando para ello adecuación de acceso y adecuación de objetivos.

Además, utilizan el Plan de Adecuaciones Curriculares Individuales (PACl), el que mencionan como un procedimiento de evaluación diferenciada, y el Diseño Universal del Aprendizaje (DUA), 
http://doi.org/10.15359/ree.25-3.22

http://www.una.ac.cr/educare

educare@una.ac.cr

enfoque curricular desarrollado originalmente por el Center for Applied Special Technology (CAS), en USA (Pastor et al., 2011), cuya propuesta intenta dotar de mayor flexibilidad al currículo, a los medios de enseñanza y a los materiales de aprendizaje. Tanto el PACI como el DUA ya han sido adoptados en varias escuelas regulares del país.

Algunas de las respuestas que ilustran esta dimensión, pueden visualizarse en la Tabla 4.

Tabla 4: Procedimientos de evaluación diferenciada utilizados por educadores diferenciales en sus prácticas evaluativas en estudiantes con discapacidad intelectual integrados en escuelas regulares

Participante Contenido declarado

Participante 2 En relación a la evaluación diferenciada en los alumnos que tienen Discapacidad Intelectual que yo atiendo, trato de incorporar diferentes formas de evaluar En esto caso por ejemplo, evaluaciones formales sumativas directamente relacionadas con los objetivos de aprendizaje que se trabajó durante la clase de matemáticas o lenguaje, pero también utilizo evaluaciones informales que yo llamaría evaluaciones de proceso, en donde, por ejemplo, yo a veces aplico tests de entrada cortitos, donde voy viendo el progreso que tienen los alumnos frente a dicho objetivo de aprendizaje para posteriormente aplicar una evaluación sumativa o una evaluación auténtica. Entonces, la evaluación diferenciada, en mi caso, yo la utilizo de diferentes formas, también o hasta mediante una observación directa mediante una lista de cotejo donde yo veo si el niño va logrando o no o va progresando mediante las clases, mediante las actividades el logro de aprendizaje que yo espero.

Participante 5 Dentro de las prácticas evaluativas, en primera instancia, está la adecuación curricular, que, como dije anteriormente, se realiza en conjunto con la profesora de asignatura. Se acuerda la extensión de la evaluación, los ítems que se van a evaluar y los objetivos de aprendizajes que se acordaron en un principio que se iban evaluar para el alumno en específico. De acuerdo a eso se va modificando el instrumento original de evaluación; en el caso de ser necesario el alumno puede ser interrogado oralmente, está la flexibilidad, y todas esas decisiones se van tomando de acuerdo a las características del alumno y las características de las asignaturas en la cual se está evaluando y a la vez obviamente del contenido que se va a evaluar. Es importante mencionar también que las evaluaciones se realizan de la siguiente manera: una vez de que son visadas por un jefe de departamento del establecimiento, ellos lo vuelven a enviar al profesor de asignatura, es ahí donde se produce esta articulación en la que se realiza la adecuación curricular para el alumno.

Nota: Elaboración propia.

\section{Instrumentos utilizados por los educadores y las educadoras diferenciales, para evaluar los aprendizajes del estudiantado con discapacidad intelectual que asisten a PIE en escuelas comunes de enseñanza}

En relación con los instrumentos de evaluación, en tanto que medios a través de los cuales el personal docente obtiene la información que se requiere del nivel de aprendizaje 
http://doi.org/10.15359/ree.25-3.22

del estudiantado, las educadoras y los educadores diferenciales entrevistados señalan que estos instrumentos de evaluación deben ser coherentes con las adecuaciones curriculares o planificaciones diseñadas especialmente para estudiantes con DI que asisten a los PIE en escuelas comunes de enseñanza.

Una parte del grupo entrevistado señala utilizar instrumentos adaptados o adecuados a las necesidades educativas especiales del estudiantado con DI. Estos instrumentos responden a una planificación previa de la evaluación y son, en ese sentido, coherentes con ella. Las principales adecuaciones o adaptaciones a las evaluaciones son: ampliar la letra de los textos, ejemplificar ejercicios, incorporar imágenes para facilitar la comprensión de instrucciones, utilizar regletas con fórmulas que permiten resolver problemas en la asignatura de matemáticas, mencionar los pasos a seguir para desarrollar un ejercicio, eliminar algunos ítems o número de ejercicios en las pruebas o guías de trabajo, realizar segmentación de la tarea y evaluar de manera oral.

Llama la atención que, pese a su formación especializada, el profesorado especial entrevistado señala que, para evaluar los aprendizajes de sus escolares con NEE derivadas de Dl, utilizan el mismo instrumento de evaluación que el profesorado de enseñanza básica utiliza para estudiantes sin DI: adapto la prueba del curso (participante 3), utilizo los mismos instrumentos de evaluación que utilizan en el curso (Participante 5), hago casi lo mismo que la profesora básica, pero con las adecuaciones pertinentes (Participante 10). Algunas de las respuestas que ilustran esta dimensión pueden visualizarse en la Tabla 5.

Tabla 5: Instrumentos utilizados por personal educativo diferencial para estudiantes que presentan discapacidad intelectual que asisten a PIE en escuelas comunes de enseñanza

\begin{tabular}{cl}
\hline Participante & \multicolumn{1}{c}{ Contenido declarado } \\
\hline Participante 1 & $\begin{array}{l}\text { Sí, mayormente son esos los que utilizo: interrogaciones orales, exposiciones... es según como mejor } \\
\text { el alumno pueda expresarse, como se le facilite más... }\end{array}$ \\
Participante 3 & $\begin{array}{l}\text { Los instrumentos que más yo aplico son pruebas escritas cortas; adapto la prueba del curso según } \\
\text { las necesidades del estudiante. También utilizo pautas de cotejo, ya que es importante saber cómo } \\
\text { van los niños, les hago hacer disertaciones, trabajos escritos y algunas veces interrogaciones orales; } \\
\text { eso es lo que hago con ellos. }\end{array}$ \\
Participante 5 & $\begin{array}{l}\text { Utilizo los mismos instrumentos de evaluación que utilizan en el curso, solo realizo adecuaciones } \\
\text { tales como: agregar ilustraciones, ampliar la letra, eliminar cantidad de items, ejemplificar, etc. }\end{array}$ \\
Participante 10 & $\begin{array}{l}\text { Hago desde pruebas mixtas hasta rúbricas para evaluar trabajos, hago casi lo mismo que la Profesora } \\
\text { Básica, pero con las adecuaciones pertinentes, además que todas las adecuaciones que se hacen son } \\
\text { en acuerdo al PACly con el equipo de aula, son parte del trabajo colaborativo entre docentes... }\end{array}$ \\
\hline
\end{tabular}

Nota: Elaboración propia. 
http://doi.org/10.15359/ree.25-3.22

http://www.una.ac.cr/educare

educare@una.ac.cr

\section{Conclusiones}

Este trabajo de investigación se planteó como objetivo principal conocer las prácticas evaluativas diferenciadas del profesorado de educación especial destinadas a escolares que presentan DI y que asisten a aulas integradas en escuelas regulares de enseñanza, a través de los PIE. Asimismo, interesó analizar los significados que el profesorado de educación especial participante del estudio otorga a la evaluación diferenciada.

Al respecto, se concluye que la mayoría del profesorado especial entrevistado utiliza tipos, procedimientos e instrumentos de evaluación diferenciada para escolares que presentan DI, alineado con las propuestas teóricas de la evaluación para la educación general. En ese sentido, llama la atención que el estudiantado con DI esté siendo sometido en los establecimientos regulares de enseñanza que participaron del estudio a una evaluación preponderantemente de tipo tradicional, basada en el currículo y en forma de pruebas de papel y lápiz. Esto respondería más bien a criterios de homogeneización y se basaría en prácticas de enseñanza y de evaluación referidas exclusivamente al aprendizaje de contenidos transmitidos y repetidos durante las clases. Esto puede ser interpretado como un recurso adaptativo, es decir como una forma de profesionales de la educación especial de plegarse a los mandatos de la educación básica regular, recurso adaptativo que les permitiría poder funcionar dentro de un sistema escolar común, en un espacio educativo común. Al respecto, podríamos hipotetizar que la educación especial no ha logrado aún, después de 10 años de inclusión en la educación regular, funcionar con las prácticas de enseñanza y evaluación que le son propias y que, seguramente, tal como lo indican Sagredo-Lillo et al. (2020) respecto a que la gestión del tiempo para el trabajo colaborativo en el sentido que es uno de los aspectos determinantes para el desarrollo de la labor educativa inclusiva, el grupo de profesionales entrevistado no cuenta con espacios de reflexión suficientes acerca del ejercicio de su profesión.

Lo anterior dejaría entrever el desarrollo de una fase aún muy inicial de inclusión educativa de escolares con discapacidad intelectual en los establecimientos de educación regular. En efecto, solo una parte de los docentes y las docentes señala utilizar procedimientos de evaluación diferenciada de acuerdo con adecuaciones curriculares, para lo cual utilizan los dos grandes tipos: de acceso y de objetivos.

Asimismo, es interesante observar que no aparecen en los discursos de quienes participaron del estudio la alusión a prácticas evaluativas relacionadas con la evaluación auténtica, tales como la evaluación alternativa o performance assessment, ni a la evaluación basada en problemas, así como tampoco a la evaluación formativa. Esto llama la atención, pues estas constituyen prácticas que destacan su relación con la aplicación de una determinada habilidad aprendida en contexto escolar al contexto de la vida real.

En efecto, tal como fue señalado por Spooner et al. (2017), toda la niñez con discapacidad debe aprender, además de habilidades académicas, habilidades de la vida diaria, a través de prácticas pedagógicas basadas en evidencias, como la evaluación especializada con demostración empírica que la investigación en la disciplina ha proporcionado. La educación 
http://doi.org/10.15359/ree.25-3.22

basada en evidencia identifica a todos aquellos enfoques de enseñanza que se han mostrado efectivos por cuerpos fiables de investigación (Courtade et.al., 2014) y tiene como objetivo priorizar la enseñanza y la evaluación de aprendizajes de calidad.

De esta forma, conforme a los resultados de este estudio y a que la aplicación de prácticas identificadas como efectivas sobre la base de investigaciones de alta calidad produce mejores resultados de aprendizaje (Cook et al., 2012), desde el punto de vista práctico se sugiere incluir, en la formación inicial docente, contenidos de enseñanza y evaluación especializada basada en evidencia científica, de manera que el estudiantado con discapacidad intelectual que asiste a establecimientos regulares de enseñanza reciba una educación de la mejor calidad posible de parte del personal educativo especialista.

Asimismo, y para avanzar hacia sistemas educativos con una inclusión con estándares de calidad, es necesario que los centros realicen un análisis reflexivo de los procesos de enseñanza y prácticas de evaluación que llevan a cabo, con el fin de establecer mejoras en sus dinámicas curriculares y organizativas (Escarbajal Frutos et al., 2017).

Por otra parte, desde el punto de vista de la investigación en la especialidad, es necesario fomentar los estudios en enseñanza y evaluación de aprendizajes orientados a escolares con discapacidad intelectual que asisten a centros regulares de enseñanza, tanto de las perspectivas cualitativa como cuantitativa, para poder abordar mejor y de manera más amplia, el fenómeno en estudio.

\section{Declaración de Material complementario}

Este artículo tiene disponible, como material complementario:

-La versión preprint del artículo en https://doi.org/10.5281/zenodo.4067022

\section{Referencias}

Agostini Martins, M. de F., Kaplan, C. V. y Sipes, M. L. (2018). Caminos y prácticas evaluativas en la educación especial: Experiencias en Brasil y Argentina. Pro-Posições,, 29(1). 20-36 https:// doi.org/10.1590/1980-6248-2017-0116

Arnaiz Sánchez, P., de Haro Rodríguez, R. y Guirao Lavela, J. M. (2015). La evaluación en educación primaria como punto de partida para el desarrollo de planes de mejora inclusivos en la región de Murcia. Revista Electrónica Interuniversitaria de Formación del Profesorado, 18(1), 103-122. https://doi.org/10.6018/reifop.18.1.214351

Barría Rojas, S. y Jurado de los S., P. (2016). El perfil profesional y las necesidades de formación del profesor que atiende a los alumnos con discapacidad intelectual en la formación laboral. Profesorado. Revista de Curriculum y Formación del Profesorado, 20(1), 287-310. https:// recyt.fecyt.es/index.php/profesorado/article/view/49880 
http://doi.org/10.15359/ree.25-3.22

http://www.una.ac.cr/educare

educare@una.ac.cr

Cajigas, R. y García, Y. M. (2014). La evaluación de los aprendizajes en las ciencias naturales: Una compilación bibliográfica (2000-2013) [Tesis de licenciatura]. Universidad del Valle. https://bibliotecadigital.univalle.edu.co/bitstream/handle/10893/7601/3467-0473442. pdf;jsessionid=4414E2A065EA40B957B988EA4E02AB53? sequence $=1$

Calderón Bravo, K. (2011). Evaluación diferenciada: Discursos y prácticas de los docentes de enseñanza básica, en tres establecimientos educacionales de la corporación municipal de La Florida. Revista Electrónica Diálogos Educativos, 11(22) 139-182. https://dialnet.unirioja. es/ejemplar/303832

Castillo Arredondo, S. y Cabrerizo Diago, D. (2003). Prácticas de evaluación educativa: Materiales e instrumentos. Pearson-Prentice Hall.

Cook, B. G., Smith, G. J. y Tanskerley, M. (2012). Evidence-based practices in education. En K. R. Harris, S. Graham y T. Urdan (Eds.), APA educational psychology handbook (Vol. 1, pp. 495528). American Psychological Association.

Courtade, G. R., Test, D. W. y Cook, B. B. (2014). Evidence-based practices for learners with severe intellectual disability. Research and Practice for Persons with Severe Disabilities, 39(4) 305318. https://doi.org/10.1177/1540796914566711

de Vries, W. (2015). Las múltiples facetas de la evaluación educativa. Revista Mexicana de Investigación Educativa. 20(66), 679-683. http://www.scielo.org.mx/scielo.php?script=sci arttext\&pid=S1405-66662015000300001

Escarbajal Frutos, A., Arnáiz Sánchez, P. y Giménez Gualdo, A. (2017). Evaluación de las fortalezas y debilidades del proceso educativo en centros de infantil, primaria y secundaria desde una perspectiva inclusiva. Revista Complutense de Educación, 28(2), 427-443. http://dx.doi. org/10.5209/rev_RCED.2017.v28.n2.49423

Fernández Núñez, L. (2006). ¿Cómo analizar datos cualitativos? Butlletí La Recerca. (Ficha 7), 1-13. https://es.scribd.com/document/205939052/Fernandez-Lissette-Como-Analizar-DatosCualitativos

Hammersley, M. y Atkinson, P. (1994). Etnografía. Métodos de investigación. Paidós.

Jara Henríquez, M. y Jara Coatt, P. (2018). Concepciones y prácticas evaluativas declaradas por los docentes en respuesta a las necesidades educativas especiales de carácter permanente. Revista Latinoamericana de Educación Inclusiva, 12(2), 59-77. https://dx.doi.org/10.4067/ $\underline{\text { S0718-73782018000200059 }}$

Krippendorff, K. (1990). Metodología de análisis de contenido: Teoría y práctica. Paidós.

Ministerio de Educación de Chile (1997). Decreto 511. Aprueba Reglamento de Evaluación y Promoción Escolar de niños y niñas de Enseñanza Básica. https://www.bcn.cl/leychile/ navegar?idNorma $=71532$ 
http://doi.org/10.15359/ree.25-3.22

Ministerio de Educación de Chile (2005). Nueva Política de Educación Especial. Autor. https:// especial.mineduc.cl/normativa/politica-nacional-educacion-especial/

Ministerio de Educación de Chile (2009). Decreto con toma de razón Nº 0170 Santiago -14/05/2009. Fija normas para determinar los alumnos con necesidades educativas especiales que serán beneficiarios de las subvenciones para Educación Especial. Autor. http://especial.mineduc. cl/wp-content/uploads/sites/31/2016/08/201304231500550.DEC200900170.pdf

Ministerio de Educación de Chile. (2016). Unidad de Educación Especial. Autor. https://especial. mineduc.cl/hola-mundo/

Muñoz Vega, E. F. (2006). Talleres para la construcción de instrumentos evaluativos en educación. Editorial Bibliográfica Internacional.

Pastor, C. A., Sánchez Serrano, J. M. y Zubillaga del Río, A. (2011). Diseño universal para el aprendizaje (DUA). Pautas para su introducción en el currículo. https://www.educadua.es/ doc/dua/dua pautas intro cv.pdf

Román, M. y Murillo, F. J. (2013). Evaluación en el campo educativo: Del sentido a la práctica. Revista Iberoamericana de Evaluación Educativa, 6(1), 7-12. https://revistas.uam.es/index. php/riee/article/view/3837/4022

Rueda Beltrán, M. (2010). La recuperación de la evaluación educativa. Perfiles Educativos, 32(128), 3-7. http://www.perfileseducativos.unam.mx/iisue_pe/index.php/perfiles/ article/view/18904/17938

Sagredo-Lillo, E. J., Bizama Muñoz, M. P. y Careaga Butter, M. (2020). Gestión del tiempo, trabajo colaborativo docente e inclusión educativa. Revista Colombiana de Educación, (78), 343360. http://doi.org/10.17227/rce.num78-9526

Sandín Esteban, M. P. (2003). Investigación cualitativa en educación: Fundamentos y tradiciones. McGrawHill.

Santibáñez Riquelme, J.D. (2001). Manual para la evaluación del aprendizajeestudiantil:Conceptos, procedimientos, análisis e interpretación para el proceso evaluativo. Trillas.

Spooner, F., McKissick, B. R. y Knight, V. F. (2017). Establishing the state of affairs for evidencebased practices in students with severe disabilities. Research and Practice for Persons with Severe Disabilities, 42(1) 8-18. https://doi.org/10.1177/1540796916684896

Stake, R. E. (2010). Investigación cualitativa: El estudio de cómo funcionan las cosas. The Guilford Press.

Tójar Hurtado, J. C. (2006). Investigación cualitativa: Comprender y actuar. La Muralla. 